\title{
Human Amniotic Membrane: Hope in Periodontal Regeneration
}

\author{
Dr. Megha Phogat Rana ${ }^{1}$, Dr. Nidhi Mehrotra ${ }^{2}$ \\ ${ }^{1}$ Reader, Department of Periodontology, Seema Dental College and Hospital, Rishikesh \\ ${ }^{2}$ Post Graduate, Department of Periodontology, Seema Dental College and Hospital, Rishikesh
}

\begin{abstract}
Several treatment modalities have been applied over the years to achieve periodontal regeneration with no predictable outcome. However, in the emerging field of regenerative medicine, the mesenchymal stem cells derived from human fetal membrane have shown promising results. The amniotic sac encloses the developing foetus through gestation and is composed of amnion and chorion membranes. The clinical application of these membranes not only maintains the structural and anatomical configuration of regenerated tissues, but also contributes to the enhancement of healing through reduction of post-operative scarring and providing a rich source of stem cells. Periodontal regeneration using amniotic membrane provides excellent predictability, improved long-term outcomes, and superior aesthetics over other treatment options.
\end{abstract}

Keywords: aesthetics, amnion, healing, mesenchymal stem cells, periodontal regeneration

\section{Introduction}

Periodontal disease is a chronic inflammatory condition that occurs in response to predominantly Gram negative bacterial infection originating from dental plaque.[1] The ultimate goal of the periodontal reconstructive surgery is to restore the supporting tissues lost as a sequel of inflammatory periodontal disease. Different modalities have been used in the past for the achievement of this goal which includes procedures like scaling, root planing, soft tissue curettage, and flap surgeries.[2] These procedures are used along with regenerative techniques like bone grafting, guided tissue regeneration, derivatives and substitutes of bone and biologic factors like enamel matrix proteins; but the outcomes are not predictable and are associated with high degree of variability.[1] With manifold developments in our understanding of periodontal regeneration, biologic and material sciences, complete regeneration still is an unrealistic situation in many clinical conditions due to complexity of biological events, factors and cells involved in the regenerative process of the periodontium.[3]

Stem cell therapy is emerging as a powerful tool to generate biological substitutes and regenerate the damaged tissue with high proliferability, differentiability and function. Many efforts are in progress to develop novel bioengineered products and include involvement of mesenchymal stem cells (MSCs). Recently, the fetal derived mesenchymal cells (MSCs) from the placenta or other gestational tissues like the amniotic fluid, umbilical cord have emerged as novel materials rich in stem cells. [3]

Applications of amnion membrane include chemical or thermal burns, correction of corneal epithelial defects, neurotrophic corneal ulcers, leaking blebs after glaucoma surgery, reconstruction of conjunctival and ocular surfaces, ocular cicatricial pemphigoid or Stevens-Johnson syndrome, and bullous keratopathy. These membranes have also been used in furcation defects, intrabony defects, and gingival recession coverage.[2] In this review, the various properties and uses of the placental membranes are discussed.

\section{History}

The use of placental tissue for the treatment of wound started more than 100 years ago when Davis in 1910 first used these fetal membranes as skin substitutes for treating open wounds.[3] Sabella and Stern in 1913 described its use for burnt and ulcerated skin surfaces.[2],[4] They evaluated the accelerative effect of the membrane on epithelialization and the reduction in pain in the wound. In 1940, De Röth first reported use of fetal membranes in the ocular surface. He used fresh amnion and chorion as a biological dressing material for management of conjunctival defects. [2]

With no ideal and practical means of preparation, sterilization and storage available at that time, the use of these membranes was very limited.[3] Kim and Tseng in 1995 gave the preservation method for maximal maintenance of biologic properties of membranes.[2] In 1965, Dino et al demonstrated for the first time that amniotic membrane could be separated, sterilized and safely used at a later date. Since then a lot of research has been initiated to understand the true regenerative potential of this membrane. [3]

The utilization of amniotic membrane waned in the early 1980 's because of increase in the communicable diseases such as H.I.V./A.I.D.S., Hepatitis, etc. Amnion re-appeared in the cryopreserved form for the treatment of ophthalmic wounds in the late 1990's and early 2000's.[4] Lawson in 1985 was the first who studied the use of amniotic membrane along with pectoralis major muscle for oral cavity reconstruction. He concluded that placement of amnion over the deep aspect of the muscle that is exposed to the oral cavity resulted in a more rapid development of mucosa. When muscle was used without amniotic membrane, the healing process usually took twice as long. Also, when amnion was not used, it showed a significant amount of wound contracture.[5]

Amniotic membrane (AM) is now used successfully in the treatment of burns; creation of biologic surgical dressing; 


\section{International Journal of Science and Research (IJSR) ISSN (Online): 2319-7064 \\ Index Copernicus Value (2013): 6.14 | Impact Factor (2015): 6.391}

reconstruction of the oral cavity, bladder, and vagina; tympanoplasty; arthroplasty; abdominal surgery; and corneal transplantation.[6] It has also been introduced as a suitable membrane for vestibuloplasty in dentistry.[7]

\section{Structure and Composition of Human Amniotic Membrane}

The amniotic membrane (AM) encases the amniotic fluid and fetus, and is highly flexible because of which it is easily be separated from the chorion.[1],[3] AM has two types of cells with different embryological origins: amnion epithelial cells derived from embryonic ectoderm and amnion mesenchymal cells from embryonic mesoderm. At ultra structural level AM is a thin, tough, transparent, avascular composite membrane composed of three major layers: a single epithelial layer, a thick basement membrane, and an avascular mesenchyme consisting mainly of collagen[3] (Figure 1).

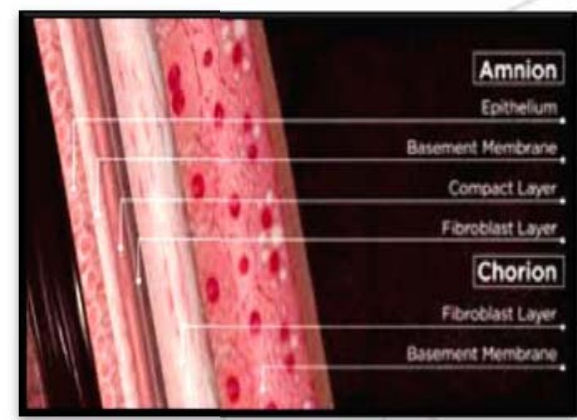

Figure 1: Structure of Human Placenta

The amniotic epithelial cell layer is a single layer of flat, cuboidal and columnar cells that are in direct contact with the amniotic fluid. It is from this layer that amniotic MSC (AMSC) are isolated and stored to be used for regenerating tissues.[3] The epithelial cells secrete collagen type III and IV and non collagenous glycoproteins like laminins, nidogen, fibronectin and vitronectin within the basement membrane that serve as adhesion ligands transmitting signals and interacting at cell surface receptors.[8] There are no nerves, muscles, or lymphatics in the amniotic membrane.[3] These human amniotic epithelial and mesenchymal cells (HAE \& HAM cells) express pluripotency and are potent stem cells reservoirs.[9] The amniotic epithelium has a specialized arrangement of intracellular cytoskeletal filaments such as actin, $\alpha$-actinin, spectrin, ezrin, cytokeratins, vimentin, and desmoplakin indicating their role in the structural integrity and modulation of cell shape of the healing tissue.[10] The amniotic mesoderm layer consists of macrophages and fibroblast-like mesenchymal cells.[3]

The basement membrane of the amnion is very similar to the basement membrane found in the other parts of the body like the conjunctiva or gingiva.[1] Basement membrane consists of collagen type IV and VII, laminin 1 and 5, fibronectin, and basic fibroblast growth factor (bFGF).[11] Laminin-5 being the most prevalent in the amnion membrane helps in cellular adhesion of gingival cells, invasive growth of fibroblasts and angiogenesis in the early phases of wound healing.[3] The side of amniotic tissue whose subchains are identified as having conjunction of collagen IV with laminin is especially effective in facilitating epithelial cell adhesion.[12] The basal lamina of AM contains a large amount of proteoglycans like heparan sulfate that is one of the major proteoglycan in the gingiva.[3]

The AM stroma contains mitogenic factors, antiangiogenic factors, anti-inflammatory proteins, and natural protease inhibitors and anti scarring properties that allow the wound to heal in a much faster and efficient way.[13] The matrix of human AM contains abundant growth factors like keratinocyte growth factor (KGF), basic-fibroblast growth factor (b-FGF), transforming growth factor-beta (TGF- $\beta$ ), nidogen growth factor (NGF), and epidermal derived growth factor (EDGF) which promote periodontal regeneration. In addition, the presence of integrin a6/b4 as the main ligands in the basement membrane which participates in the construction of the hemidesmosome like structure favours the adhesion and anchoring of ESCs to the healing wound. This facilitates migration of epithelial cells, reinforces adhesion of basal epithelial cells, promotes epithelial differentiation, and prevents apoptosis.[3] Table 1 describes the structure and composition of the amniotic membrane.

Table 1: Structure and composition of Amnion[2]

\begin{tabular}{|c|c|}
\hline Structure & Composition \\
\hline Epithelium & Single layer of flat cuboidal columnar cells \\
\hline $\begin{array}{c}\text { Basement } \\
\text { Membrane }\end{array}$ & $\begin{array}{c}\text { Collagen types III, IV and V, fibronectin, } \\
\text { laminin and nidogen }\end{array}$ \\
\hline Compact layer & Collagen types I, III, IV, V, VI and fibronectin \\
\hline Fibroblast layer & $\begin{array}{c}\text { Collagen types I, III and VI, fibronectin, laminin } \\
\text { and nidogen }\end{array}$ \\
\hline
\end{tabular}

Various stem cell markers such as octamer-binding transcription factor (OCT) - 4, hepatocytes nuclear factor-3 $\beta$ (HNF-3 $\beta$ ), nestin and nanog which are specific stem cells markers are also found in the amnion membrane.[14,15] The stem cell markers like epidermal marker CA125 and general epithelial markers such as cytokeratins and vimentin are present in large amount in the amniotic epithelial cells.[16,17] These MSCs have the ability to accelerate the inflammatory phase towards the proliferative phase which is critical for treating chronic wounds like periodontitis.[3] The mechanism of accelerated wound healing by amnion membrane is shown in Figure 2.

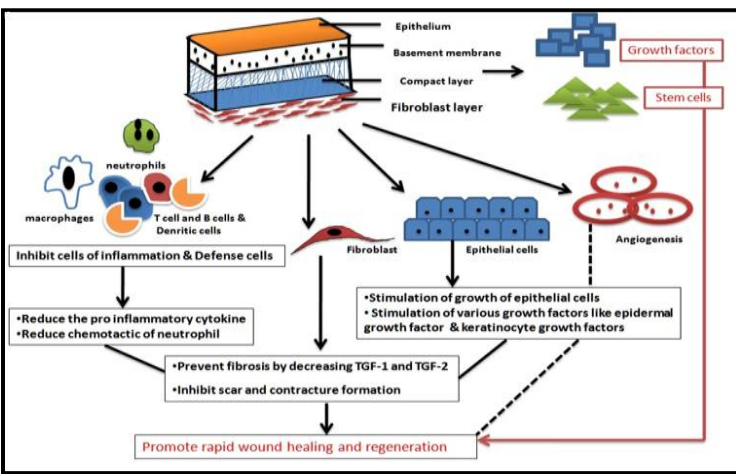

Figure 2: Mechanism of wound healing by amniotic membrane [3] 


\section{International Journal of Science and Research (IJSR) \\ ISSN (Online): 2319-7064 \\ Index Copernicus Value (2013): 6.14 | Impact Factor (2015): 6.391}

\section{Properties}

\section{Anti inflammatory}

The Mesenchymal Stem Cells (MSCs) in the AM decrease the secretion of proinflammatory cytokines like Tumor Necrosis Factor alpha (TNF- $\alpha$ ) and Interferon (IFN) while increasing the production of anti-inflammatory cytokines interleukin-10 and interleukin-4.[1],[4] The proinflammatory mediators, interleukin-1 $\alpha$ and interleukin- $1 \beta$, are also suppressed by matrix of stroma of amniotic membrane. The inhibitors of matrix metalloproteinases found in the amniotic membrane decreases matrix metalloproteinases (MMPs) released by infiltrating neutrophils and macrophages.[2] Various tissue inhibitors of metalloproteinases 1, 2, 3, and 4, interleukin-10, and interleukin-1 receptor antagonists and endostatin which inhibit endothelial cell proliferation, angiogenesis, and tumor growth are also expressed by human amniotic epithelial and mesenchymal cells.[18] AM also reduces the recruitment of various other inflammatory cells including polymorphonuclear cells, CD3 cells, CD4 T cells and CD11b cells to the injured site thereby reducing the inflammation.[1]

\section{Angiogenesis}

The cells of the AM enhance the production of Vascular Endothelial Growth Factor (VEGF) byh activating the VEGF receptors 1 and 2. Extensive neovascularization after the application of AM is due to the release of angiogenic factor like insulin derived growth factor (IGF) that promotes granulation tissue formation and epithelialization.[3] The increase capillary blood flow to the lyophilized amniotic membranes when used as graft material in vestibuloplasty promotes healing.[19]

\section{Immunomodulatory}

The unique molecular surface architecture and biochemical properties of AM that is derived from the layer of trophoblast cells renders it unsusceptible to maternal immune attack.[1],[3] The native amniotic epithelial cells (AECs) express the non-polymorphic, non-classical human leukocyte antigen (HLA-G) but lack the polymorphic antigens HLA-A, B (Class IA) and HLA-DR (Class II) on their surfaces. The class I antigen is seen in almost all cells of the amniotic membrane unlike the class II antigen which is only present in some fibroblasts. These mesenchymal stem cells are different from other nucleated mammalian cells as they show little allogenic reactivity when administered to MHC unmatched adult immune competent recipients.[3] The cells of AM neither express the programmed cell death receptor 1 (PD1) (an inhibitory receptor that is normally expressed on activated $\mathrm{T}$ and $\mathrm{B}$ cells), nor its two ligands: programmed death ligands 1 and 2 (PD-L1 and PD-L2) and the immunoglobulin-like transcript receptors 2, 3, and 4 (ILT R-2, ILT R-3, and ILT $\mathrm{R}-4)$.[1] The immunomodulatory action of AM is also due to lack of expression of co-stimulatory cell surface molecules such as CD80, CD-86, and CD-40 in the presence or absence of Interferon- $\Upsilon$ (IFN-Y). Furthermore, they actively suppress $\mathrm{T}$ cell, dendritic cell and B cell function that downmodulate exuberant inflammation. [3]

\section{Antimicrobial and Antiviral}

Amniotic membrane firmly adheres with the wound via fibrin and elastin linkages that seals the wound and prevent contamination.[1] This tight adherence helps in restoring lymphatic integrity, protects circulating phagocytes from exposure and allows faster removal of surface debris and bacteria from the wound.[20] The antimicrobial activity of the MSCs in AM is mediated by two mechanisms: directly via the secretion of antimicrobial factors such as LL-37 [21] and indirectly via secretion of immunomodulative factors which will upregulates bacterial killing and phagocytosis by immune cells.[22] Many bactericidal products of purine metabolism and lysozyme are also found in the AM. Defensins, mostly beta 3 defensins, secretary leukocyte proteinase inhibitor (SLPI) and elafin present in the amniotic cells act as components of the innate immune system to provide protection from infection.[3] Its antiviral properties are exhibited by the presence of a powerful antiviral agent, Cystatin E which is an analogue of cysteine proteinase inhibitors.[2],[3]

\section{Anti Scarring}

AM secretes vascular endothelial growth factor (VEGF), hepatocyte growth factor (HGF) that maintains a proper balance between TGF-1 and TGF-3 that prevents scarring. It also down-regulates TGF-beta and its receptor expression by fibroblast that reduces fibrosis at the site. This also modulates the healing of a wound by promoting tissue reconstruction.[1],[3] Various immune cells like $\mathrm{T}$ cell, dendritic cell and B cell are also actively suppressed that prevents pathological remodeling and excessive fibrosis.[1]

\section{Promotion of Epithelialization}

Amniotic membrane facilitates migration of epithelial cells, reinforces basal cell adhesion, promotes epithelial differentiation, prevents epithelial apoptosis, and promotes epithelialization in healing of wounds. It can also promote expansion and maintenance of epithelial progenitor cells in vivo and can produce endothelin-1 and parathyroid hormone related protein. Brain natriuretic peptide and corticotrophin releasing hormone are also produced by membrane epithelial cells which play roles in increasing cellular proliferation and calcium metabolism. The basement membrane of AM serves as a safe and suitable bed for the growth of epithelial cells. Sufficient oxygenation for epithelial cells is provided by its good permeability in contrast to other synthetic materials. Thus, amniotic membrane is an ideal tissue which facilitates the growth of epithelial cells, helping in their migration and differentiation.[2]

\section{Reduction of Pain}

Amnion membrane (AM) reduces pain as it diminishes inflammation and provides a better state of hydration that soothes the wound bed to promote faster healing. The soft mucoid lining of amniotic membrane also protects the exposed nerve endings from external irritant that help to decrease pain sensation.[1],[3]

\section{Increased extracellular matrix deposition}

MSCs differentiation helps in regenerating the damaged tissue and regulates the local cellular responses to injury by paracrine signaling. It helps in cell survival, proliferation, migration and gene expression of epithelial cells, endothelial 


\section{International Journal of Science and Research (IJSR) ISSN (Online): 2319-7064 \\ Index Copernicus Value (2013): 6.14 | Impact Factor (2015): 6.391}

cells, keratinocytes, and fibroblasts. MSC-conditioned medium acts as a chemoattractant for macrophages, endothelial cells, epidermal keratinocytes, and fibroblasts which accelerate wound closure.[3]

\section{Processing of amniotic membrane}

For clinical use, amniotic membrane can be prepared in the following forms:[1]

- Fresh membrane

- Dried membrane

- Frozen membrane

- Freeze derived irradiated membrane

- Stabilized amniotic membrane

- Cryopreserved membrane

\section{Advantages of amniotic stem cells over bone marrow stem cells}

1. The recovery of MSCs from the amniotic membrane is a non-invasive procedure.

2. Bone marrow mesenchymal stem cell and progenitor cells unlike AM mesenchymal cells show decreasing proliferative potential with increasing age.

3. AM-isolated cells undergo in vitro osteogenic, adipogenic, chondrogenic and myogenin expression along with CD34 and von willebrand factor which permits unrestricted use, distribution and reproduction of these MSC in culture medium.

4. The amniotic cells can proliferate without any need of a second cell type serving as a feeder layer as they create their own feeder layer. The basal layer that attaches to the culture dish may play the role of an autologous feeder layer, serving as a substrate for attachment or possibly providing secreted factors that help induce or maintain undifferentiated Amniotic epithelial cells (AECs).

5. The average yield is more than 100 million AECs per amnion which is much higher than those collected from the bone marrow.[3]

\section{Uses and Applications of Amniotic Membrane}

The amniotic membrane is a composite membrane consisting of a pluripotent cellular element embedded in a semipermeable membranous structure. Amniotic membrane is an immunotolerant structure and the pluripotent stem cells possess the ability of transdifferentiation to other cellular elements of the periodontium making it a suitable candidate for guided tissue regeneration (GTR). Excellent revascularization of the amniotic membrane is another favorable property of this natural structure.[23] Gomes et al in 2001 studied the use of amnion grafts to line the floors of cortical bone defects and to cover the superficial surface of the defects. At 90 days, amnion tissue was in direct apposition to newly formed bone. At 120 days, the amnion tissue grafts were no longer present and bone had completely filled the defects. The authors concluded that the use of amnion tissue grafts did not inhibit repair in guided bone regeneration and may have been beneficial for its antibacterial properties.[24]
Amniotic membrane can be used for transplantation either as a temporary graft or as a permanent graft. It can be used alone or in conjunction with other surgical procedures when employed as permanent graft. In temporary grafting procedure, it is sutured to both healthy host tissue and site of interest at the same time as a bandage or dressing, or patch. This is so done to promote healing of host epithelial lining lying underneath. The membrane is invariably dissolved once epithelialization is complete. The removal is carried out in a period varying from 2 to 6 weeks. When used for permanent grafting, for example, in cornea or conjunctiva, it is sutured to fill in the tissue defect so that host cells proliferate into it and a sound integration with the host tissue is achieved.[2]

The AM can be used either alone with only amniotic epithelium (intact AM) or without it (denuded AM); Cryopreserved, freeze dried or in hyperdried form. It can also be used in powder form in the areas of suture lines and hard to cover areas. These fetal membranes are being used as a graft or dressing in the management of burns; alternative treatment to manage wounds in the oral cavity like the tongue, buccal mucosa, vestibule, palatal mucosa, and floor of the mouth; in the reconstruction of the oral cavity, bladder, and vagina; tympanoplasty; arthroplasty etc.[1] Good biocompatibility and mechanical properties like permeability, stability, elasticity, flexibility, plasticity, and resorbability also makes it a promising scaffolding material in tissue engineering as in cell adhesion and the potential for delivery of biomodulatory agents such as growth factors and genetic materials.[1],[3] The physical properties of amniotic membrane have proven it to be compatible with corneal surface of the eye. The eye and placental membranes have so much similarity in their immune modulatory properties that they have been referred to as "parallel universes".[2] These membranes are extremely thin around $300 \mathrm{~nm}$ in cross sectional thickness unlike the other collagen membranes used for guided tissue regeneration which are around 700 $800 \mathrm{~nm}$.[25] Because of its thin diameter it intimately molds according to the defect anatomy and root surfaces easily.[3]

Periodontal reconstructive surgery consists of various mucogingival procedures aiming to benefit periodontal health by reconstruction of lost hard and soft tissues. Variety of techniques have been employed for root coverage for preventing additional loss and enhancing the esthetics. Newer techniques allow root coverage with more promising results by using amniotic membrane. $\mathrm{H}$. Singh and $\mathrm{H}$. Singh in 2013 presented a case report on bioactive amniotic membrane as a membrane for the treatment of isolated gingival recession. The results showed significant root coverage with uneventful healing.[26] Shetty et al in 2014 compared usage of Platelet-rich Fibrin (PRF) and amniotic membrane in bilaterally occurring multiple Miller Class I recession. $100 \%$ root coverage was observed with both of the membranes but the results were stable even after seven months in the amniotic membrane-treated site.[23]

Antiinflammatory and antiscarring property of AM have shown decreased necrosis and rapid healing of ulcers with herpes simplex virus (HSV), varicella zoster virus-infected tissues, erythema multiforme major (Stevens-Johnson syndrome) and cervical necrotizing fasciitis. HAM has been 


\section{International Journal of Science and Research (IJSR) \\ ISSN (Online): 2319-7064}

Index Copernicus Value (2013): 6.14 | Impact Factor (2015): 6.391

tried in the reconstruction of TMJ ankylosis as it prevents fibrosis and reankylosis when used as an interpositional material.[3] AM is even used as a carrier for local delivery of the various drugs like antibiotic netilmycin (NTM) and antiviral drugs like acyclovir (ACV) and trifluridine (TFU).[2],[3] Amnion has been tried as a graft material after vestibuloplasty where it prevents secondary contraction after the surgery and maintains the postoperative vestibular depth.[3] Samandari et al in 2004 suggested that the amniotic membrane graft might be used as a potential graft material for vestibuloplasty.[19] Kothari et al in 2012 also concluded that grafts of amniotic membrane are viable and reliable for covering of the raw surface, prevent secondary contraction after vestibuloplasty, and maintain the postoperative vestibular depth.[7]

Human amniotic membrane is also used as a potential dressing that accomplishes four major goals: haemostasis, reduction of water loss through evaporation, and providing a moist environment for cell survival and growth, acting as barrier to microbial colonization and pain reduction.[2] Arai et al in 2011 showed the clinical usefulness of the hyper-dry amniotic membrane as an intraoral wound dressing material. The results suggested that it is biologically acceptable to oral wounds and could be a suitable clinical alternative for the repair of the oral mucosa.[27] The graft of amniotic membrane is a viable and reliable method to cover the exposed periosteum as they serve as a good alternative to mucosal and skin grafts.[3]

\section{Limitations of Amniotic Membrane}

The use of amniotic membranes requires skill; thus, operator's inexperience is a limitation. There is always an associated risk of infection transmission with transplantation of amniotic membranes. Adequate precautions should be taken and safety criteria should be included in application of these biological membranes. Amniotic membranes are fragile membranes, so they need to be dealt with very carefully. Cryopreserved/hyperdry membranes are expensive. The procedure associated with use of these membranes is technique-sensitive and also depends on defect morphology.[2]

\section{Conclusion}

The use of this novel biological membrane is rising in various fields of tissue engineering, medicine, regeneration biology and stem cell research. The clinical application of amniotic membrane not only maintains the structural and anatomical configuration of regenerated tissues, but also contributes to the enhancement of healing through reduction of post-operative scarring and subsequent loss of function and providing a rich source of stem cells. However, further research and long term clinical trials investigating the full potential of this stem cell reservoir are still warranted to strengthen the fact amniotic membrane is indeed a reservoir for regeneration.

\section{References}

[1] Mishra S and Singh S. Human amniotic membrane: Can it be a ray of hope in periodontal regeneration? Indian $\mathrm{J}$ Res 2014;3(9):118-21.

[2] Gupta A, Kedige SD, Jain K. Amnion and chorion membranes: potential stem cell reservoir with wide applications in peridontics. Int J Biomater 2015;1-9.

[3] Chopra A and Thomas BS. Amniotic membrane: A novel material for regeneration and repair. J Biomim Biomater Tissue Eng 2013;18(1):106-13.

[4] Gupta I, Gupta R, Gokhale ST, Sharma A. Placental tissues: fixing smiles. Int J Innov Scientific Res 2014;7(1):57-62.

[5] Sharma Y, Maria A, Kaur P. Effectiveness of human amnion as a graft material in lower anterior ridge vestibuloplasty: A clinical study. J Maxillofac Oral Surg 2011;10(4):283-7.

[6] Kiany F and Moloudi F. Amnion membrane as a novel barrier in the treatment of intrabony defects: A controlled clinical trial. Int J Oral Maxiilofac Implants 2015;30:639-47.

[7] Kothari CR, Goudar G, Hallur N, Sikkerimath B, Gudi $\mathrm{S}$, Kothari MC. Use of amnion as a graft material in vestibuloplasty: A clinical study. Br J Oral Maxillofac Surg 2012;50:545-49.

[8] Sniadecki NJ, Desai RA, Ruiz SA, Chen CS Nanotechnology for cell-substrate interactions. Ann Biomed Eng 2006;34:59-74.

[9] Parolini O, Alviano F, Bagnara GP, Bilic G, Buhring HJ, Evangelista $M$ et al. Concise review: isolation and characterization of cells from human term placenta: outcome of the first international Workshop on Placenta Derived Stem Cells. Stem Cells 2008;26:300-11.

[10]Bani-Yaghoub M, Wilson P, Hengstschlager M, Nikaido T. Amniotic Stem Cells: Potential in Regenerative Medicine. Stem Cells International 2012;26-32.

[11]Rinastiti M, Harijadi, Santoso ALS, Sosroseno W. Histological evaluation of rabbit gingival wound healing transplanted with human amniotic membrane. Int J Oral Maxillofac Surg 2006;35:247-51.

[12] Samandari MH, Adibi S, Khoshzaban A, Aghazadeh S, Dihimi P, Torbaghan SS et al. Human amniotic membrane, best healing accelerator, and the choice of bone induction for vestibuloplasty technique (an animal study). Transplant Res Risk Management2001;3:1-8.

[13] Boudreau N, Sympson CJ, Werb Z, Bissell MJ. Suppression of ICE and apoptosis in mammary epithelial cells by extracellular matrix. Science 1995;267:891-3.

[14] Kubo M, Sonoda Y, Muramatsu R, Usui M. Immunogenicity of human amniotic membrane in experimental xenotransplantation. Invest Ophthalmol Vis Sci 2001;42:1539-46.

[15] Witkowska-Zimny M, Walenko K. Stem cells from adipose tissue. Cell Mol Biol Lett 2001;16:236-57.

[16] Nanbu Y, Fujii S, Konishi I, Nonogaki H, Mori T (1989) CA 125 in the epithelium closely related to the embryonic ectoderm: the periderm and the amnion. Am J Obstet Gynecol 1989;161:462-7.

[17]Beham A, Denk H, Desoye G. The distribution of intermediate filament proteins, actin and desmoplakins 
in human placental tissues as revealed by polyclonal and monoclonal antibodies. Placenta 1988;9:479-92.

[18] Haо Y, Ma DHK, Hwang DG, Kim DS, Zhang F. Identification of antiangiogenic and antiinflammatory proteins in human amniotic membrane. Cornea 2000;19(3):348-52.

[19] Samandari MH, Yaghmaei M, Ejlali M, Moshref M, Saffar AS. Use of amnion as a graft material in vestibuloplasty: a preliminary report. Oral Surg Oral Med Oral Pathol Oral Radiol Endod 2004;97:574-8.

[20] Rao TV and Chandrasekhram V. Use of dry human and bovine amnion as a biological dressing. Arch Surg 1981;116:891-6.

[21] Krasnodembskaya A, Song Y, Fang X, Gupta N, Serikov V, Lee JW et al. Antibacterial effect of human mesenchymal stem cells is mediated in part from secretion of the antimicrobial peptide LL-37. Stem cells 2010;28:2229-38.

[22] Talmi YP, Sigler L, Inge E, Finkelstein Y, Zohar Y. Antibacterial properties of human amniotic membranes. Placenta 1991;12:285-8.

[23] Shetty SS, Chatterjee A, Bose S. Bilateral multiple recession coverage with platelet rich fibrin in comparison with amniotic membrane. Indian J Soc Periodontol 2014;18(1):102-6.

[24] Gomes MF, dos Anjos MJD, Nogueira TD, Guimaraes SAC. Histologic evaluation of the osteoinductive property of autogenous demineralized dentin matrix on surgical bone defects in rabbit skulls using human amniotic membrane for guided bone regeneration. Intl J Oral Maxillofac Implants 2001;16(4):563-71.

[25] Rothamel D, Schwarz F, Sager M, Herten M, Sculean A, Becker J. Biodegradation of differently cross-linked collagen membranes: an experimental study in the rat. Clin Oral Implants Res 2005;16:369-78.

[26] Singh H and Singh H. Bioactive amnion as a guided tissue regeneration (GTR) membrane for treatment of isolated gingival recession. A case report. Indian J Dentistry 2013;4(2):110-3.

[27] Arai N, Tsuno H, Okabe M, Yoshida T, Koike C, Noguchi $\mathrm{M}$ et al. Clinical application of a hyperdry amniotic membrane on surgical defects of the oral mucosa. J Oral Maxillofac Surg 2012;70(9):2221-8.

\section{Author Profile}

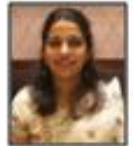

Dr. Megha Phogat Rana is reader in the Department of Periodontology, Seema Dental College and Hospital, Rishikesh. Graduated in 2006 from DAV Yamunanagar and completed post graduation in 2010 from BBD College of Dental Sciences, Lucknow.

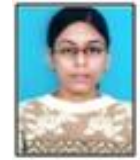

Dr. Nidhi Mehrotra is Post graduate student in the Department of Periodontology, Seema Dental College and Hospital, Rishikesh. Graduated in 2011 from Rama Dental College, Kanpur 